\title{
The Salvia miltiorrhiza monomer IH764-3 induces apoptosis of hepatic stellate cells in vivo in a bile duct ligation-induced model of liver fibrosis
}

\author{
LEI LIU $^{1}$, JUAN WEI ${ }^{2}$, XIAOXIA HUO ${ }^{3}$, SHUMING FANG ${ }^{1}$, DONGMEI YAO ${ }^{1}$, \\ JUNPING GAO $^{1}$, HUIQING JIANG ${ }^{1}$ and XIAOLAN ZHANG ${ }^{1}$ \\ ${ }^{1}$ Department of Gastroenterology, The Second Hospital of Hebei Medical University, \\ Hebei Key Laboratory of Gastroenterology, Hebei Institute of Gastroenterology, Hebei 050000; \\ ${ }^{2}$ Department of Hepatology, The Fifth Hospital of Shijiazhuang City, Hebei 050021; \\ ${ }^{3}$ Department of Gastroenterology, The Third Hospital of Shijiazhuang City, Hebei 050011, P.R. China
}

Received March 26, 2012; Accepted August 6, 2012

DOI: $10.3892 / \mathrm{mmr} .2012 .1076$

\begin{abstract}
During the process of liver fibrosis, hepatic stellate cells (HSCs) play a critical role in the excessive production of extracellular matrix (ECM). Previous studies have indicated that the monomer IH764-3, one of the major bioactive components of Salvia miltiorrhiza, is able to inhibit HSC proliferation and induce the apoptosis of activated HSCs in vitro. In the current study, we used a rat model of liver fibrosis induced by bile duct ligation (BDL) to investigate the effect of the monomer IH764-3 on the induction of apoptosis in HSCs in vivo. The rat model of liver fibrosis was established by BDL. Immunohistochemical staining of $\alpha$-smooth muscle actin ( $\alpha$-SMA) was performed to detect HSC activation and proliferation and HSC apoptosis was detected by terminal deoxynucleotidyl transferase dUTP nick end labeling (TUNEL) and $\alpha$-SMA immunohistochemical double staining. In addition, the protein expression levels of focal adhesion kinase (FAK), p-FAK (Tyr397), extracellular signal-regulated kinase (ERK) and p-ERK and the mRNA expression levels of FAK and ERK were measured by western blotting and reverse transcription-polymerase chain reaction (RT-PCR), respectively. The monomer IH764-3 was associated with a significant decrease in intrahepatic fibrogenesis and collagen
\end{abstract}

Correspondence to: Professor Xiaolan Zhang, Department of Gastroenterology, The Second Hospital of Hebei Medical University, Hebei Key Laboratory of Gastroenterology, Hebei Institute of Gastroenterology, No. 215 Heping West Road, Shijiazhuang, Hebei 050000, P.R. China

E-mail: xiaolanzh@126.com

Abbreviations: BDL, bile duct ligation; HSC, hepatic stellate cells; $\alpha$-SMA, $\alpha$-smooth muscle actin; TUNEL, terminal deoxynucleotidyl transferase UTP-nick end labeling; FAK, focal adhesion kinase; ERK, extracellular signal-regulated kinase

Key words: Salvia miltiorrhiza monomer IH764-3, liver fibrosis, hepatic stellate cells, proliferation, apoptosis deposition and attenuated the liver fibrosis induced by BDL. Immunohistochemical staining revealed that the expression of $\alpha$-SMA in the IH764-3 group was significantly decreased compared with that in the model group (12.92 \pm 2.45 vs. $22.65 \pm 2.16 \%, \mathrm{P}<0.01)$. TUNEL and $\alpha$-SMA immunohistochemical double staining also confirmed that IH764-3 increased the apoptotic rate of the activated HSCs $(34.8 \pm 4.5$ vs. $4.72 \pm 0.37 \%, \mathrm{P}<0.01)$. Moreover, the results revealed that IH764-3 downregulated the expression levels of FAK, p-FAK (Tyr397), ERK and p-ERK in the liver tissue of rats with liver fibrosis. The monomer IH764-3 ameliorates experimental liver fibrosis by inhibiting HSC proliferation and inducing HSC apoptosis, warranting its use as a potential therapeutic agent in the treatment of liver fibrosis.

\section{Introduction}

Liver fibrosis is a common consequence of chronic liver injury that is induced by a variety of etiological factors that lead to liver cirrhosis. This progressive pathological process is characterized by the accumulation of extracellular matrix (ECM) proteins (1-3). The activation and proliferation of hepatic stellate cells (HSCs) has been shown to promote the excessive production and secretion of ECM and therefore to play a central role in liver fibrogenesis (4). Moreover, the activated HSCs are eliminated mainly through apoptosis, since it is difficult for them to return to quiescence $(5,6)$, making them an appealing target for the treatment of liver fibrosis.

Increased attention has been paid to the antifibrotic activity of natural herbs, in particular Salvia miltiorrhiza, a traditional Chinese herbal medicine that has a variety of pharmacological effects, including anti-inflammatory, oxygen free radical-removing and antioxidant activities $(7,8)$. In order to explore the antifibrotic mechanism of Salvia miltiorrhiza, the monomer IH764-3, a major potent component extracted from Salvia miltiorrhiza, was investigated. In our previous study, we demonstrated that the monomer IH764-3 inhibited proliferation and induced apoptosis in HSCs stimulated by $\mathrm{H}_{2} \mathrm{O}_{2}$ in vitro (9-11). Moreover, it increased the ratio of matrix 
metalloproteinase (MMP)-13 to tissue inhibitor of MMP (TIMP)-1 in HSCs by downregulating the expression of focal adhesion kinase (FAK) and extracellular signal-regulated kinase (ERK) in vitro (12). However, there is no direct evidence that the monomer IH764-3 has a potential role in the treatment of liver fibrosis.

The purpose of the present study was to investigate the impact of the monomer IH764-3 on the proliferation and apoptosis of HSCs in a rat model of liver fibrosis induced by bile duct ligation (BDL) in vivo.

\section{Materials and methods}

Reagents. The Salvia miltiorrhiza monomer IH764-3 was donated by Professor Yang Chunzheng from the Hematology Institute of the Chinese Academy of Medical Sciences. The mouse anti- $\alpha$-smooth muscle actin ( $\alpha$-SMA) monoclonal antibody, rabbit anti-FAK and p-FAK (Tyr397) polyclonal antibodies and rabbit anti-ERK and mouse anti-p-ERK monoclonal antibodies were purchased from Santa Cruz Biotechnology, Inc. (Santa Cruz, CA, USA). The streptavidin peroxidase (SP) immunohistochemical kit was acquired from Zhongshan Biotechnology Corporation (Beijing, China). The terminal deoxynucleotidyl transferase dUTP nick end labeling (TUNEL) detection kit was purchased from Boster Biotechnology, Inc. (Wuhan, China). The reverse transcriptionpolymerase chain reaction (RT-PCR) amplification system was purchased from Promega Biotechnology, Inc. (Madison, WI, USA).

Animal models. A total of 40 adult male Sprague-Dawley rats weighing 350-450 g were obtained from the Experimental Animal Center of Hebei Medical University (Certificate No. 04037). The rats were randomly divided into three groups: a control group $(n=10)$, a model group $(n=15)$ and a IH764-3 group $(n=15)$. All rats were housed in plastic cages with free access to clean-grade food and water and received human care. The experiments were performed in compliance with the national ethical guidelines for the care and use of laboratory animals.

A rat model of hepatic fibrosis was established by common BDL as previously described (13). All rats undergoing laparotomy were injected intraperitoneally with $100 \mathrm{ml} / \mathrm{kg}$ ketamine hydrochloride and chloral hydrate to induce deep anesthesia. In the model and IH764-3 groups, the peritoneal cavity was opened and the common bile duct was completely ligated (double-ligated) with 3-0 silk and cut between the ligatures. In the control group, animals underwent a sham surgery that consisted of exposure but no ligation of the common bile duct. All surgeries were performed under aseptic conditions. The monomer IH764-3 was diluted to $8 \mathrm{~g} / \mathrm{l}$ with saline. On the day of the surgery, the rats in the IH764-3 group were injected intraperitoneally with IH764-3 (40 mg/kg/day) and the rats in the control and model groups were administered normal saline. Post-operatively, at 14 days, all animals were anesthetized, sacrificed and their livers harvested. The liver tissue specimens were fixed in $4 \%$ phosphate-buffered paraformaldehyde (Shijiazhuang Huarui Scientific and Technological Co., Ltd., Shijiazhuang, China) and stained with hematoxylin and eosin (H\&E), Masson's trichrome (MT) and immunohistochemical staining. Other specimens were snap-frozen in liquid nitrogen and stored at $-80^{\circ} \mathrm{C}$ for the analysis of mRNA and proteins.

Histopathology. The liver specimens were fixed for 12-24 h in $4 \%$ phosphate-buffered paraformaldehyde and then embedded in paraffin for light microscopic examination. Tissue sections (5 $\mu \mathrm{m}$ thick) were stained with H\&E for morphological evaluation and MT staining for assessment of the degree of fibrosis. The collagen expression levels were measured using a Motic Med 6.0 digital video-image analysis system (Motic China Group Co., Ltd, Xiamen, China) and are expressed as optical density values.

Immunohistochemical detection of $\alpha$-SMA. All immunohistochemical studies were performed on $5-\mu \mathrm{m}$ thick, paraformaldehyde-fixed, paraffin-embedded liver tissue sections using the SP technique. The sections were deparaffinized in xylene, rehydrated in graded ethanol and rinsed three times (5-min washes) with $0.01 \mathrm{M}$ phosphate-buffered saline (PBS). Endogenous peroxidase activity was quenched with $3 \%$ hydrogen peroxide in methanol for $30 \mathrm{~min}$ at room temperature. All subsequent washings of the sections were with PBS (three changes, 5 min each). Following antigen retrieval using $0.1 \mathrm{M}$ citrate buffer $(\mathrm{pH}$ 6.0) in a microwave oven at $98^{\circ} \mathrm{C}(20 \mathrm{~min})$, the sections were immediately cooled to room temperature and then blocked with $10 \%$ goat serum at $37^{\circ} \mathrm{C}$ for $30 \mathrm{~min}$. Superfluous goat serum was blotted with a piece of filter paper and the sections were then incubated overnight at $4^{\circ} \mathrm{C}$ with the primary antibody (mouse anti- $\alpha$-SMA monoclonal antibody) at a dilution of 1:100. After washing the sections, the biotin-conjugated rabbit anti-mouse secondary antibody (1:100 dilution) was added and the sections were incubated at $37^{\circ} \mathrm{C}$ for $30 \mathrm{~min}$. Sections were washed and then incubated with streptavidin-peroxidase complex (1:200 dilution) at $37^{\circ} \mathrm{C}$ for $30 \mathrm{~min}$. Following another wash, diaminobenzidine solution was used as a substrate for peroxidase, yielding a brown-colored positive reaction. The negative control samples were processed under the same conditions, except that normal mouse serum (1:100 dilution) was used in place of the primary antibody. The $\alpha$-SMA-positive expression levels were measured using a Motic Med 6.0 digital video-image analysis system and are expressed as optical density values.

TUNEL and $\alpha$-SMA immunohistochemical double staining. The sections were deparaffinized in xylene, rehydrated in graded ethanol and rinsed three times (5-min washes) with $0.01 \mathrm{M}$ PBS. The sections were then incubated with $3 \%$ acetic acid ( $\mathrm{pH} \mathrm{2.5)} \mathrm{for} 10 \mathrm{~min}$ at room temperature, rinsed three times (2-min washes) with distilled water (DW) and digested with protease $\mathrm{K}$ at $37^{\circ} \mathrm{C}$ for $10 \mathrm{~min}$. The sections were rinsed three times (2-min washes) in triethanolamine-buffered saline (TBS) and, following the addition of the TUNEL reaction mixture, were incubated overnight at $4^{\circ} \mathrm{C}$. The sections were then washed and blocked with confining liquid at $37^{\circ} \mathrm{C}$ for $30 \mathrm{~min}$. The biotin-conjugated anti-digoxin antibody (Digibind; 1:100 dilution) was added and the sections were incubated at $37^{\circ} \mathrm{C}$ for $30 \mathrm{~min}$. The sections were washed and then incubated with SABC-AP (1:100 dilution) at $37^{\circ} \mathrm{C}$ for $30 \mathrm{~min}$. Following another wash, the sections were stained 
with BCIP/NBT. The immunohistochemical staining of $\alpha$-SMA was then detected according to the preceding method. The negative control samples were processed under the same conditions, with the exception of the terminal deoxynucleotidyl transferase labeling. The nuclei of the apoptotic HSCs were stained blue-black by BCIP/NBT in the TUNEL assay and the cytoplasms of the activated HSCs were stained brown by DAB in the $\alpha$-SMA immunohistochemical staining assay. Thus, the cells with brown cytoplasms and blue-black nuclei were apoptotic activated HSCs. The apoptotic rate was determined from the ratio of the area of apoptotic activated HSCs to the total area of activated HSCs.

Preparation of hepatic tissue protein and western blots. Approximately $100 \mathrm{mg}$ rat liver tissue was collected, rinsed twice with ice-cold PBS, homogenized and transferred into an Eppendorf tube. Cytoplasmic proteins were then extracted using modified RIPA lysis buffer [50 $\mathrm{mmol} / 1$ Tris- $\mathrm{HCl}$ (pH 7.5), $100 \mathrm{mmol} / 1 \mathrm{NaCl}, 1 \% \mathrm{NP}-40,0.5 \%$ sodium deoxycholate, $2 \mu \mathrm{g} / \mathrm{ml}$ Leupeptin, $1 \%$ SDS, 2 mmol/1 EDTA, $1 \mathrm{mmol} / \mathrm{l} \mathrm{PMSF}, 50 \mathrm{mmol} / \mathrm{l} \mathrm{HEPES}$ and $100 \mathrm{mmol} / 1$ sodium orthovanadate]. The protein concentrations were determined by Coomassie Brilliant Blue staining. The proteins, fractionated by 8 or $10 \%$ SDS-PAGE, were transferred to a nitrocellulose filter membrane. After blocking, the membrane was incubated with rabbit anti-FAK polyclonal antibody (1:500), rabbit anti-p-FAK (Tyr397) polyclonal antibody (1:150), rabbit anti-ERK polyclonal antibody (1:500), mouse anti-p-ERK monoclonal antibody (1:200) and goat anti- $\beta$-actin polyclonal antibody $(1: 300)$ overnight at $4^{\circ} \mathrm{C}$. The membranes were incubated at room temperature for $2 \mathrm{~h}$ with horseradish peroxidase-conjugated goat anti-rabbit IgG secondary antibody (1:5000), goat anti-mouse IgG antibody (1:3000) and rabbit anti-goat IgG antibody (1:5000). The protein bands were subsequently detected by enhanced chemiluminescence (ECL). The images were procured using Kodak ID Image Analysis software. The results are presented as the ratio of the integral optical density (IOD) of the target protein to that of $\beta$-actin.

RT-PCR. The total RNA of the hepatic tissue was extracted with the TRIzol reagent (Invitrogen, Carlsbad, CA, USA) according to the manufacturer's instructions and then reverse transcribed with the oligo-dT primer. Primer express 5.0 was used to design the following primers: FAK forward primer, 5'-ACT TGG ACG CTG TAT TGG AG-3' and reverse, 5'-CTG TTG CCT GTC TTC TGG AT-3' (833 bp amplicon); ERK forward primer, 5'-GCT GAC CCT GAG CAC GAC CA-3 and reverse, 5'-CTG GTT CAT CTG TCG GAT CA-3' (451 bp amplicon); and $\beta$-actin forward primer, 5'-AGC TGA GAG GGA AAT CGT GCG-3' and reverse, 5'-GTG CCA CCA GAC AGC ACT GTG-3' (300 bp amplicon). The primers were synthesized by Saibaisheng Gene Co., Ltd. (Beijing, China).

The RT-PCR was performed using $2 \mu \mathrm{g}$ total RNA, $1 \mu \mathrm{l}$ upstream primers $(10 \mathrm{pmol} / \mu \mathrm{l}), 1 \mu \mathrm{l}$ downstream primers (10 pmol/ $\mu \mathrm{l}), 1 \mu \mathrm{l} \mathrm{dNTP}, 10 \mu \mathrm{l} 5 \mathrm{X}$ reaction buffer AMV/Tfl, $1 \mu \mathrm{l}$ AMV reverse transcriptase (5 U/ $\mu 1), 1 \mu \mathrm{l}$ RNA Tfl DNA polymerase and $2 \mu \mathrm{g}$ DNA template made up to a total volume of $50 \mu \mathrm{l}$ with DEPC water (Kangwei Corporation, Beijing, China). RT was carried out at $41^{\circ} \mathrm{C}$ for $45 \mathrm{~min}$. The
PCR conditions were as follows: initial pre-denaturation at $94^{\circ} \mathrm{C}$ for $2 \mathrm{~min}$, denaturation at $94^{\circ} \mathrm{C}$ for $40 \mathrm{sec}$, annealing at $52^{\circ} \mathrm{C}$ for $1 \mathrm{~min}$, polymerization at $72^{\circ} \mathrm{C}$ for $1.5 \mathrm{~min}$ (35 cycles) and terminal extension at $72^{\circ} \mathrm{C}$ for $10 \mathrm{~min}$. The products were resolved using $1.5 \%$ agarose gels containing $0.5 \mathrm{mg} / \mathrm{ml}$ ethidium bromide. The optical density was assayed using a gel image analysis system. The ratio of target gene to $\beta$-actin gene in each group was semi-quantitatively determined.

Statistical treatment. Measurement data are expressed as the mean \pm standard deviation (mean \pm SD). The apoptotic rate is expressed as a percentage. Analysis was carried out using SPSS 18.0 software (SPSS, Inc., Chicago, IL, USA). The comparison of mean variability among all groups was conducted by one-way ANOVA and two group comparisons were made using the LSD test. $\mathrm{P}<0.05$ was considered to indicate a statistically significant result.

\section{Results}

Established BDL-induced liver fibrosis model. The rats actively recovered from 1 to $2 \mathrm{~h}$ following BDL. The rats had light yellow urine the next day and had developed yellow coloration in the thinner parts of the skin, including the ear, and dark yellow urine on days 5-7 after the surgery. Throughout the experiment, 4 rats died in the model group and 2 rats died of internal hemorrhage caused by intraperitoneal injection of the drug in the IH764-3 group.

IH764-3 ameliorated BDL-induced liver fibrosis. H\&E and Masson's trichrome staining revealed a histologically normal phenotype in the control group. These sections appeared to have structurally intact hepatic lobules, an orderly arrangement of hepatic plates, no hepatic cell swelling, no proliferation of the bile duct and little connective tissue in the portal area.

In the model group, the liver sections revealed that the normal arrangement of hepatic plates had disappeared, the structure of the lobules was disordered and the small bile ducts of the portal area had invasively proliferated into the lobules. Further, in addition to an enlarged portal area, there was marked fibroplasia around the infiltrating small bile ducts and in the hepatic lobules. MT staining demonstrated that the collagen area density in the model group was significantly higher than in the control group $(16.72 \pm 1.15$ vs. $3.47 \pm 0.23 \%, \mathrm{P}<0.01)$ and in the IH764-3 group, although there were no marked changes in the structure of the hepatic lobules and the small bile ducts still proliferated, the collagen fibers in the portal area slightly increased in number. The collagen area density in the IH764-3 group was also markedly decreased compared with the model group $(12.60 \pm 0.95$ vs. $16.72 \pm 1.15 \%, \mathrm{P}<0.01)$.

In summary, the swelling of hepatic cells, fatty degeneration, necrosis, regeneration and fibrosis were observed in the BDL-induced rat model and treatment with IH764-3 substantially decreased the extent of liver fibrosis (Fig. 1).

IH764-3 inhibited the proliferation of HSCs. Given that $\alpha$-SMA is an activated HSC marker, immunohistochemical staining of $\alpha$-SMA was used to quantify the activation and proliferation of HSCs. In the liver tissues of the rats in the control group, $\alpha$-SMA was occasionally detected in vascular 

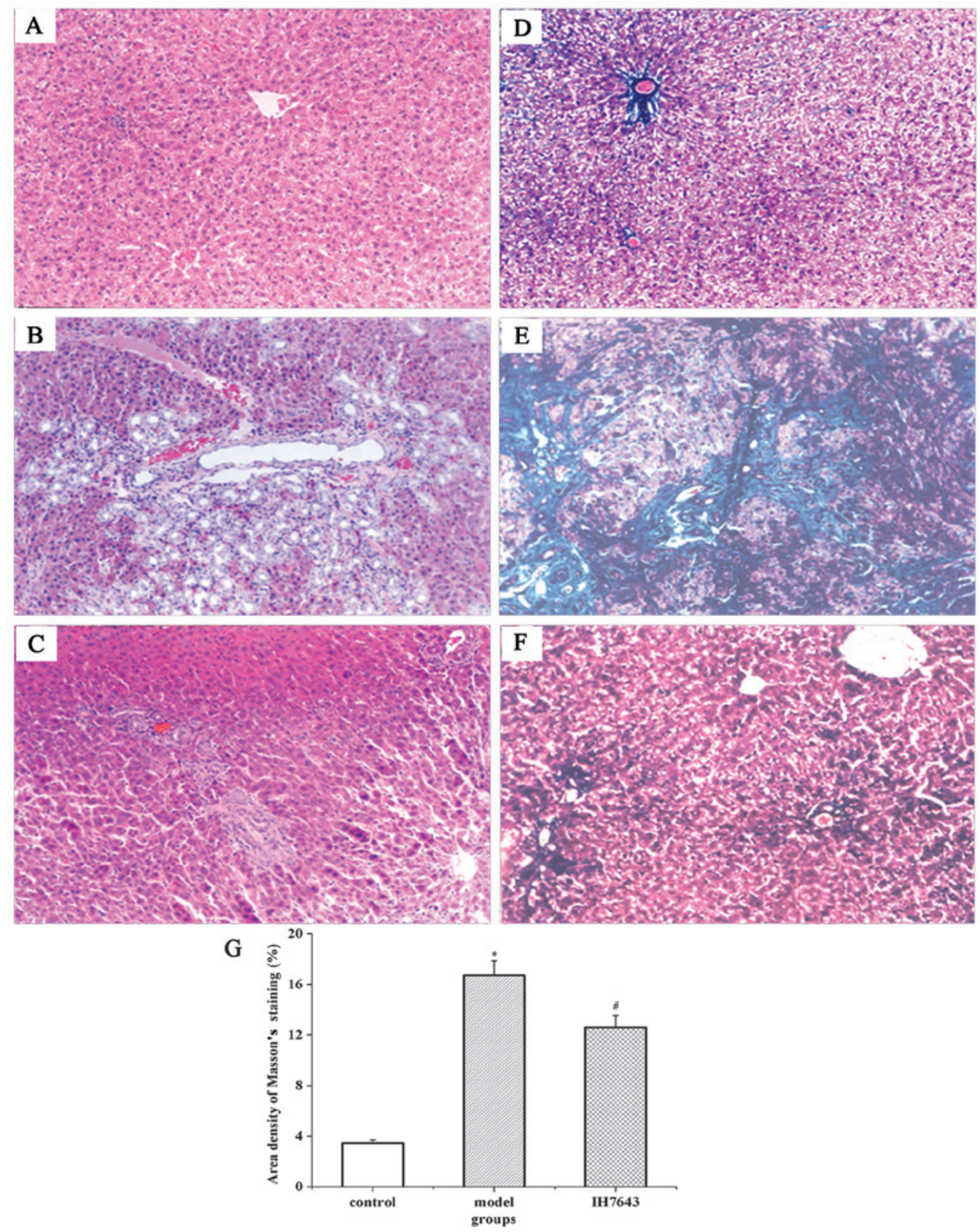

Figure 1. Effect of the monomer IH764-3 on intrahepatic fibrosis in BDL-treated rats. IH764-3 attenuated the histological damage and collagen deposition in the fibrotic model. Histological changes in the rat liver tissues were evaluated using H\&E staining. (A-C) Original magnification x100; (A) control, (B) model and (C) IH764-3 groups. Fibrotic tissues changes in the rat liver tissues were evaluated using MT staining. (D-F) Original magnification x200; (D) control, (E) model and (F) IH764-3 groups. (G) Semi-quantitative measurement of liver fibrosis in the rat liver tissues is shown. MT stain collagen area density measurements demonstrated that the collagen area density in the model group was significantly higher than in the control group. In the IH764-3 group, the collagen area density was markedly decreased compared with the model group. Data are the mean $\pm \mathrm{SD}$. $\mathrm{P}<0.01$ vs. control, " $\mathrm{P}<0.01 \mathrm{vs.}$ model. BDL, bile duct ligation H\&E, hematoxylin and eosin; MT, Masson's trichrome.

smooth muscle cells and its expression levels were low. In the model group, with the development of liver fibrosis, the $\alpha$-SMA-positive cells in the liver tissue increased significantly and were distributed mainly in the portal area, fibrous septa, hepatic sinusoids and proliferated peripheral cells of the bile duct. In the IH764-3 group, the positive expression of $\alpha$-SMA in the liver tissues of the rats was clearly decreased, particularly in the portal area and hepatic sinusoids.

The immunohistochemical results indicate that the area of $\alpha$-SMA-positive cells in the rat liver tissues of the model group was significantly increased compared with the control group $(22.65 \pm 2.16$ vs. $5.88 \pm 1.46 \%, \mathrm{P}<0.01)$ and markedly reduced in the IH764-3 group compared with the model group $(12.92 \pm 2.45$ vs. $22.65 \pm 2.16 \%, \mathrm{P}<0.01)$, but remained higher than in the control group ( $\mathrm{P}<0.01$, Fig. 2$)$.

IH764-3 induced the apoptosis of activated HSCs. HSC apoptosis was detected by TUNEL and $\alpha$-SMA immunohistochemical double staining. The nuclei of the apoptotic HSCs were colored blue-black by BCIP/NBT in the TUNEL assay, and the cytoplasms of the activated HSCs were colored brown by the immunohistochemical staining of $\alpha$-SMA. Therefore, 

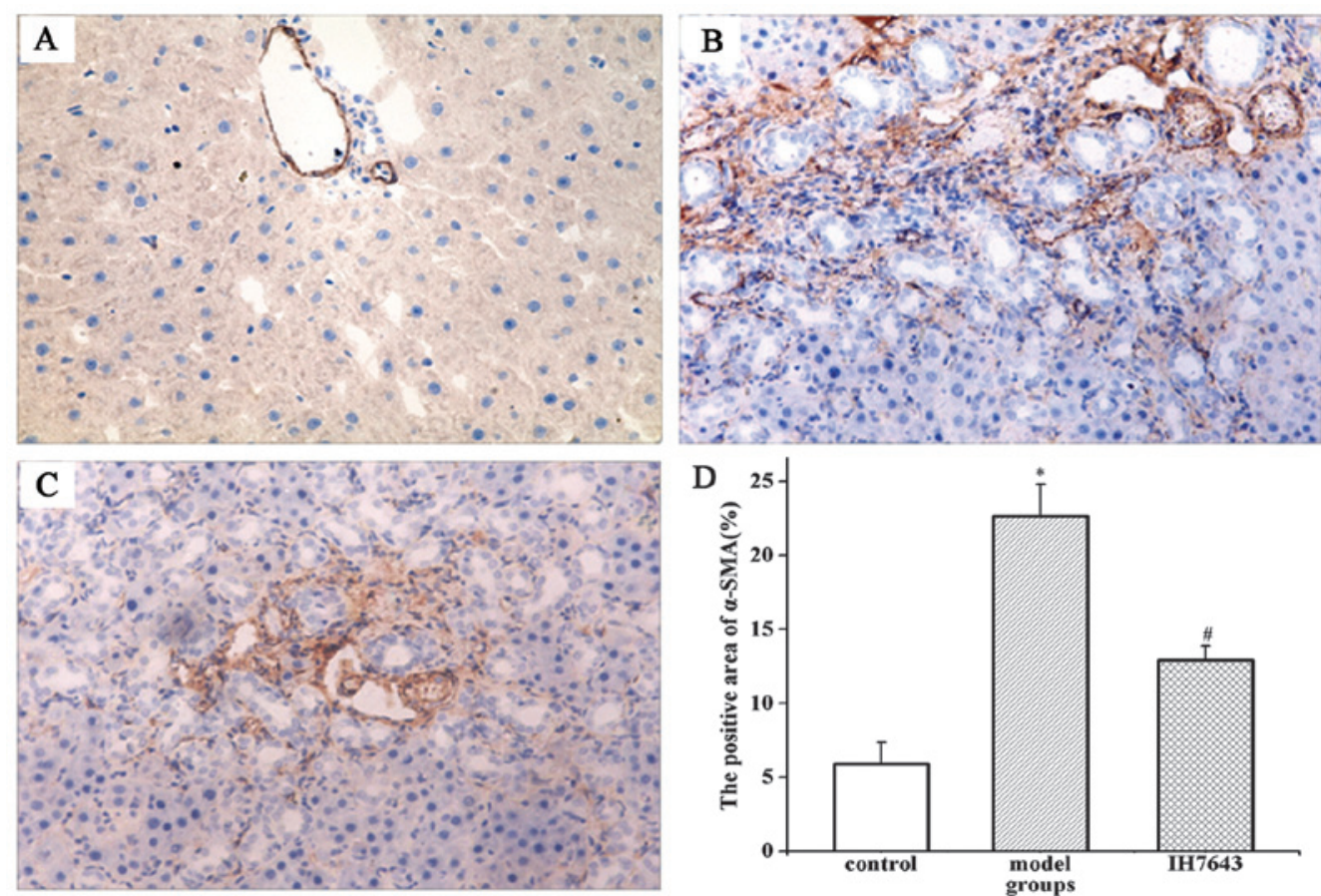

Figure 2. Monomer IH764-3 decreased $\alpha$-SMA expression in rat liver tissues. The expression levels of $\alpha$-SMA protein were analyzed by the immunohistochemical staining of rat liver tissues (A-C); original magnification x200; (A) control, (B) model and (C) IH764-3 groups. (D) The positive area of $\alpha$-SMA in the liver tissue of rats in the model group was significantly increased compared with the control group. Compared with the model group, the positive area of $\alpha$-SMA was markedly reduced in the IH764-3 group. Data are the mean \pm SD. ${ }^{~} \mathrm{P}<0.01$ vs. control, ${ }^{\prime \prime} \mathrm{P}<0.01$ vs. model. $\alpha$-SMA, $\alpha$-smooth muscle actin.
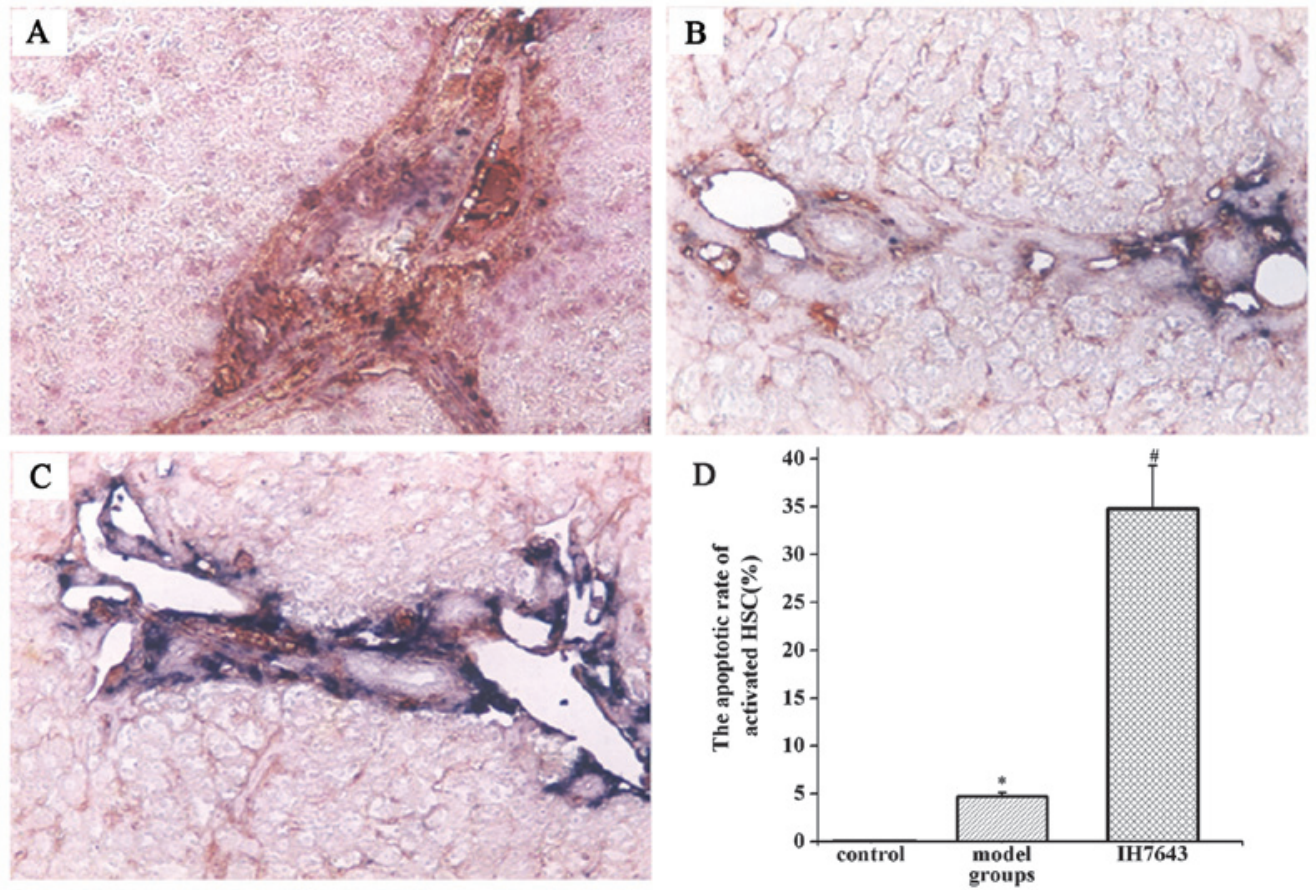

Figure 3. Monomer IH764-3 induced the apoptosis of activated HSCs. The apoptosis of activated HSCs was detected by TUNEL and $\alpha$-SMA immunohistochemical double staining in rat liver tissues (A-C); original magnification x200. (A) control, (B) model and (C) IH764-3 groups. (D) The percentage of apoptotic HSCs out of total activated HSCs (apoptotic rate of activated HSCs) in the model group was notably higher than in the control group. Compared with the model group, the apoptotic rate of activated HSCs was significantly increased in the IH764-3 group. Data are mean \pm SD. ${ }^{*} \mathrm{P}<0.01$ vs. control, ${ }^{,} \mathrm{P}<0.01$ vs. model. HSCs, hepatic stellate cells; $\alpha$-SMA, $\alpha$-smooth muscle actin.

the cells with brown cytoplasms and blue-black nuclei were apoptotic activated HSCs.

In the control group few apoptotic HSCs were detected in the rat liver tissue, and in the model group, the areas of apoptotic HSCs and activated cells increased, indicating that activation and apoptosis were concurrent in the HSCs. In the IH764-3 group, the apoptotic activated HSCs increased significantly compared with the model group. These activated 


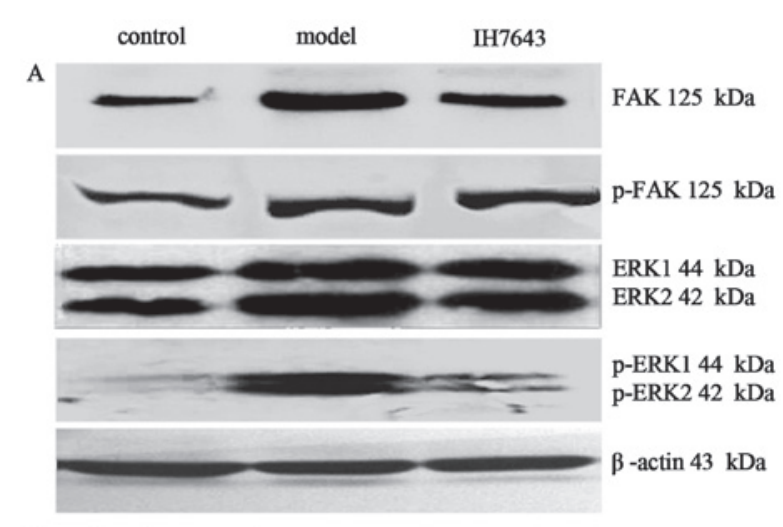

B

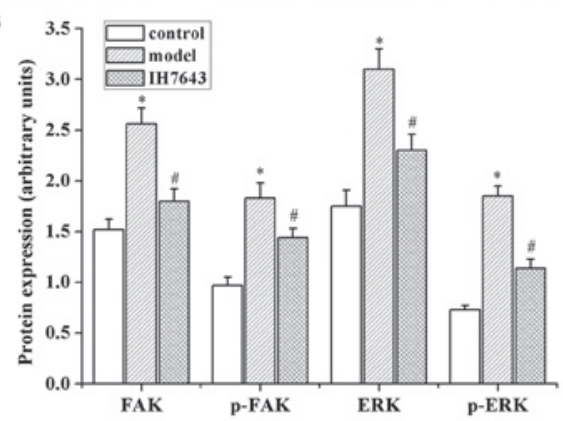

Figure 4. IH764-3 effectively inhibited the expression of FAK, p-FAK, ERK and p-ERK proteins in rat liver tissues. (A) The expression levels of FAK and ERK protein in the rat liver tissues were measured by western blotting. (B) Expression levels of the proteins were obtained by scanning densitometry and are expressed as the ratio of the IOD of the target protein to that of $\beta$-actin. The expression levels of the proteins in the model group were higher than in the control group, and were markedly reduced in the IH764-3 group compared with the model group. Data are the mean $\pm \mathrm{SD}$. ${ }^{*} \mathrm{P}<0.01$ vs. control, ${ }^{\text {P }}<<0.01$ vs. model. FAK, focal adhesion kinase; ERK, extracellular signal-regulated kinase; IOD, integral optical density.

HSCs were distributed mainly in the portal area, hepatic sinusoids and proliferated peripheral cells of the bile duct. The apoptotic rates of activated HSCs in the rat liver tissues in the control, model and IH764-3 groups were 0.01 $\pm 0.02,4.72 \pm 0.37$ and $34.8 \pm 4.5 \%$, respectively $(\mathrm{P}<0.01$; Fig. 3$)$.

IH764-3 inhibited the expression of FAK and p-FAK (Tyr397) proteins and FAK $m R N A$ in rat liver tissue. Our previous study has shown that FAK regulates HSC proliferation, and that its expression is increased during progressive liver fibrosis in BDL-treated rats $(14,15)$. Therefore, to further explore the mechanisms by which the monomer IH764-3 affects HSC apoptosis, we measured the levels of FAK and p-FAK (Tyr397).

Western blotting revealed that the protein expression levels of FAK and p-FAK (Tyr397) in the model group were higher than in the control group $(2.56 \pm 0.16$ vs. $1.52 \pm 0.10$ and $1.83 \pm 0.15$ vs. $0.97 \pm 0.10, \mathrm{P}<0.01)$ and were downregulated in the IH764-3 group compared with the model group (1.80 \pm 0.12 vs. $2.56 \pm 0.16$ and $1.44 \pm 0.99$ vs. $1.83 \pm 0.15, \mathrm{P}<0.01$, Fig. 4$)$. RT-PCR similarly demonstrated a significant increase in FAK mRNA in the model group compared with the control group $(0.83 \pm 0.06$ vs. $0.46 \pm 0.03, \mathrm{P}<0.01)$, and a marked reduction in the IH764-3 group compared with the model group $(0.69 \pm 0.04$ vs. $0.83 \pm 0.06, \mathrm{P}<0.01$; Fig. $5 \mathrm{~A}$ and $\mathrm{C}$ ).

IH764-3 inhibited the expression of ERK and $p$-ERK proteins and ERK $m R N A$ in rat liver tissue. As ERK is the

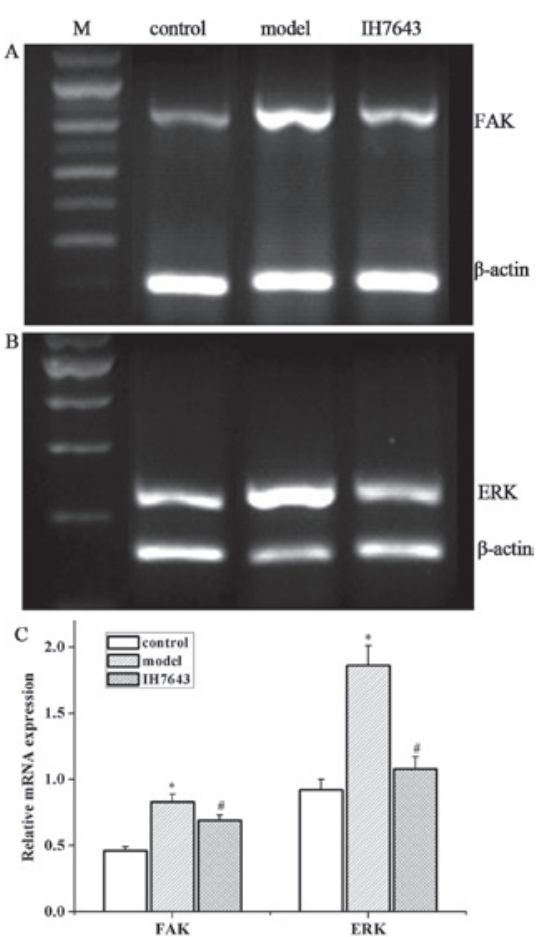

Figure 5. IH764-3 effectively inhibited the expression of FAK and ERK mRNA in rat liver tissues. RT-PCR analysis of (A) FAK and (B) ERK mRNA expression in rat liver tissues. (C) The FAK and ERK expression levels were obtained by scanning densitometry and are expressed as the ratio of the IOD of the target protein to that of $\beta$-actin. The mRNA expression levels in the model group were significantly increased compared with the control group and were markedly decreased in the IH764-3 group compared with the model group. Data are the mean $\pm \mathrm{SD} .{ }^{*} \mathrm{P}<0.01$ vs. control, ${ }^{\#} \mathrm{P}<0.01$ vs. model. FAK, focal adhesion kinase; ERK, extracellular signal-regulated kinase; RT-PCR, reverse transcription-polymerase reaction; IOD, integral optical density.

key downstream signaling molecule of FAK, we determined the expression levels of ERK and p-ERK in our study. The expression levels of ERK and p-ERK proteins were significantly increased in the model group compared with the control group $(3.10 \pm 0.20$ vs. $1.75 \pm 0.16$ and $1.85 \pm 0.10$ vs. $0.73 \pm 0.04, \mathrm{P}<0.01)$. The treatment of rats with IH764-3 significantly decreased the levels of ERK and p-ERK in liver tissue $(2.30 \pm 0.16$ vs. $3.10 \pm 0.20$ and $1.14 \pm 0.09$ vs. $1.85 \pm 0.10$, $\mathrm{P}<0.01$, Fig. 4) compared with the model group. Furthermore, the mRNA levels of ERK in the model group were higher than in the control group $(1.86 \pm 0.15$ vs. $0.92 \pm 0.08, \mathrm{P}<0.01)$, and treatment with IH764-3 reduced the mRNA level of ERK in the rat liver tissues $(1.08 \pm 0.09$ vs. $1.86 \pm 0.15, \mathrm{P}<0.01$, Fig. 5B and C).

\section{Discussion}

Liver fibrosis is considered to be reversible through the apoptosis of activated HSCs and the degradation of ECM proteins (16). However, an ideal approach for the clinical treatment of liver fibrosis is lacking. Accumulated data have shown that Salvia miltiorrhiza ameliorates liver fibrosis induced by carbon tetrachloride $\left(\mathrm{CCl}_{4}\right)$ or dimethylnitrosamine (DMN) in rats $(17,18)$. Salvia miltiorrhiza extract has also been shown to exert anti-fibrosis activity in vitro by mediating TGF- $\beta / \mathrm{Smad}$ signaling in myofibroblasts (19). Furthermore, in our earlier studies, we revealed that the monomer IH764-3, a water- 
soluble extract of Salvia miltiorrhiza, inhibits HSC activation and proliferation and induces the apoptosis of HSCs stimulated by $\mathrm{H}_{2} \mathrm{O}_{2}$ in vitro (9-11), revealing that $\mathrm{IH764-3}$ may be able to affect liver fibrosis through the regulation on HSCs, the key cells of liver fibrosis.

Cholestatic fibrosis induced by BDL in rats, which is similar to human cholestatic liver injury, is a common method for producing experimental cirrhosis and is a suitable experimental model of human liver disease. Cholestatic liver injury induced by biliary obstruction causes acute hepatocellular injury and leads to progressive fibrogenesis. Although the mechanism of cholestatic liver injury is not well understood, oxidative stress or proinflammatory cytokines play an key role in the development of hepatocellular injury and fibrogenesis (20). In the current study, we selected the rat BDL model of liver fibrosis, employing H\&E and MT staining to assess the degree of fibrosis. Notably, the monomer IH764-3 had beneficial effects on intrahepatic fibrogenesis. The administration of IH764-3 substantially decreased the extent of the hepatocyte necrosis and degeneration and collagen deposition in the liver tissues, indicating that it exhibited in vivo antifibrotic effects that mitigated the BDL-induced liver fibrogenesis.

It is known that HSCs exist in the space of Disse in a relatively small quantity, in a quiescent state and expressing no $\alpha$-SMA, and their main function is to store vitamin A (21). The quiescent HSCs are activated to myofibroblasts expressing $\alpha$-SMA when stimulated by various cytokines and inflammatory mediators, and have been shown to migrate to and proliferate in sites of liver injury $(22,23)$, synthesize ECM components and upregulate the expression levels of $\alpha$-SMA and collagen matrices (24). Hence, $\alpha$-SMA indirectly represents the degree of HSC activation and proliferation. In the normal rat livers of the control group, $\alpha$-SMA was occasionally detected in vascular smooth muscle cells, and its expression level was low, revealing that few HSCs were activated. Following BDL, the area of tissue expressing $\alpha$-SMA spread to the portal area and revealed the presence of more activated HSCs. Immunohistochemical staining demonstrated that the expression levels of $\alpha$-SMA in the model group were significantly increased. In the IH764-3 group, significantly decreased expression levels of $\alpha$-SMA, particularly in the portal area and hepatic sinusoids, were observed compared with the model group, which suggests that the monomer IH764-3 may act as an antifibrotic agent by inhibiting HSC activation and proliferation in vivo.

It has been reported that activated HSCs are eliminated mainly through apoptosis, since it is difficult for them to return to quiescence $(5,6)$. In fact, the augmentation of HSC apoptosis is known to promote the resolution of fibrosis (25-27). Hence, the induction of apoptosis in activated HSCs is a critical event in the treatment of liver fibrosis. Our previous studies have demonstrated that the monomer IH764-3 is capable of inducing HSC apoptosis activated by $\mathrm{H}_{2} \mathrm{O}_{2}$ in a dose-dependent manner in vitro (11). In the present study, we observed the effect of IH764-3 on HSC apoptosis in vivo by TUNEL and $\alpha$-SMA immunohistochemical double staining. Our results revealed that the apoptotic rate of activated HSCs in the rat liver tissues of the IH764-3 group $(34.8 \pm 4.5 \%)$ was higher than in the model group $(4.72 \pm 0.37 \%)$. These results are consistent with in vitro studies of IH764-3 and suggest that the induction of apoptosis in HSCs is one of the antifibrotic mechanisms of IH764-3.

It is well known that FAK, a non-receptor protein tyrosine kinase, is involved in various cellular processes, including adhesion, migration, proliferation and apoptosis (28-30). The MAPK signaling pathway regulates diverse cellular events including proliferation, growth, differentiation and apoptosis $(31,32)$. A number of studies have revealed that the MAPK pathway is involved in the pathogenesis of fibrosis $(33,34)$. As one of the key kinases in the MAPK pathway, ERK1 has also been shown to be implicated in the development of hepatic fibrosis $(35,36)$. FAK and ERK1 promote proliferation and migration in a variety of cells. Studies have shown that FAK activation causes a cascade reaction through the MAPK pathway. Previous studies have confirmed that the downregulation of FAK by short hairpin RNA technology or the endogenous inhibitor decreases the expression level of p-FAK (Tyr397), inhibits HSC proliferation and induces HSC apoptosis in vitro, which indicates an involvement of the FAK-ERK signal transduction pathway (37-39). We also have revealed that the expression levels of FAK and ERK1 increase during progressive liver fibrosis in BDL-treated rats and that in an in vitro experiment, the monomer IH764-3 downregulated FAK and ERK1 in HSCs stimulated by $\mathrm{H}_{2} \mathrm{O}_{2}(11-13,15)$. In the present study, we demonstrated that FAK and ERK1 were decreased at the translation and transcription levels by the monomer IH764-3, which indicates that IH764-3 inhibits the FAK-ERK signal transduction pathway and that this is the mechanism by which IH764-3 inhibits HSC proliferation and induces HSC apoptosis.

In summary, the monomer IH764-3 significantly ameliorates experimental liver fibrosis by inhibiting HSC proliferation and inducing HSC apoptosis. The relevant mechanism involves inhibition of the FAK-ERK signal transduction pathway. These results provide evidence that $\mathrm{IH764-3}$ is an attractive agent for the treatment of liver fibrosis.

\section{Acknowledgements}

This study was supported by the Department of Science and Technology of Hebei Province (09966107D) and the Traditional Chinese Medicine Drug Administration of Hebei Province (No. 2007061). The authors would like to thank the Foundations for their support.

\section{References}

1. Povero D, Busletta C, Novo E, di Bonzo LV, Cannito S, Paternostro $C$ and Parola M: Liver fibrosis: a dynamic and potentially reversible process. Histol Histopathol 25: 1075-1091, 2010.

2. Friedman SL: Mechanisms of hepatic fibrogenesis (Review). Gastroenterology 134: 1655-1669, 2008.

3. Jiao J, Friedman SL and Aloman C: Hepatic fibrosis (Review). Curr Opin Gastroenterol 25: 223-229, 2009.

4. Friedman SL: Hepatic stellate cells: protean, multifunctional, and enigmatic cells of the liver (Review). Physiol Rev 88: 125-172, 2008.

5. Lakner AM, Walling TL, McKillop IH and Schrum LW: Altered aquaporin expression and role in apoptosis during hepatic stellate cell activation. Liver Int 31: 42-51, 2011.

6. Kisseleva T and Brenner DA: Hepatic stellate cells and the reversal of fibrosis (Review). J Gastroenterol Hepatol 21: S84-S87, 2006. 
7. Moon S, Shin S, Kim S, Oh HE, Han S, Lee S and Kim K: Role of Salvia miltiorrhiza for modulation of Th2-derived cytokines in the resolution of inflammation. Immune Netw 11: 288-298, 2011.

8. You Z, Xin Y, Liu Y, Han B, Zhang L, Chen Y, Chen Y, Gu L, Gao H and Xuan Y: Protective effect of Salvia miltiorrhizae injection on $\mathrm{N}(\mathrm{G})$-nitro-d-arginine induced nitric oxide deficient and oxidative damage in rat kidney. Exp Toxicol Pathol 64: 453-458, 2012

9. Liu L, Jiang HQ and Zhang XL: The effect and mechanism of Salvia miltiorrhiza monomer IH764-3 on proliferation and collagen synthesis of hepatic stellate cells stimulated by $\mathrm{H}_{2} \mathrm{O}_{2}$. Zhongguo Ying Yong Sheng Li Xue Za Zhi 19: 78-81, 2003 (In Chinese).

10. Zhang XL, Liu L and Jiang HQ: Salvia miltiorrhiza monomer IH764-3 induces hepatic stellate cell apoptosis via caspase-3 activation. World J Gastroenterol 8: 515-519, 2002.

11. Fang SM, Li CS, An JY, Dun ZN, Yao DM, Liu L and Zhang XL: The role of extracellular signal-regulated kinase in induction of apoptosis with Salvia miltiorrhiza monomer IH764-3 in hepatic stellate cells. Zhongguo Ying Yong Sheng Li Xue Za Zhi 27: 402-406, 2011 (In Chinese).

12. Liu L, Jiang HQ, Zhang XL and Zhao DQ: Effect of Salvia miltiorrhiza monomer IH764-3 on MMP-13 and TIMP-1 by downregulating the expression of focal adhesion kinase in hepatic stellate cell stimulated by $\mathrm{H} 2 \mathrm{O} 2$. Zhongguo Ying Yong Sheng Li Xue Za Zhi 23: 482-486, 2007 (In Chinese).

13. Zhang XL, Liu JM, Yang CC, Zheng YL, Liu L, Wang ZK and Jiang HQ: Dynamic expression of extracellular signal-regulated kinase in rat liver tissue during hepatic fibrogenesis. World J Gastroenterol 12: 6376-6381, 2006.

14. Huo XX, Zhang XL, Shen JG and Wei J: FAK-related non-kinase plasmid transfection inhibited hepatic stellate cell proliferation stimulated by fibronection. Clin J Hepatol 15: 547-548, 2007.

15. Zhang XL, Huo XX, Shen JA, Wei J and Jiang HQ: Focal adhesion kinase tyrosine phosphorylation promotes rat hepatic fibrogenesis and its possible mechanism. Basic \& Clinical Medicine 27: 143-147, 2007 (In Chinese).

16. Elsharkawy AM, Oakley F and Mann DA: The role and regulation of hepatic stellate cell apoptosis in reversal of liver fibrosis (Review). Apoptosis 10: 927-939, 2005.

17. Hsu YC, Lin YL, Chiu YT, Shiao MS, Lee CY and Huang YT: Antifibrotic effects of Salvia miltiorrhiza on dimethylnitrosamine-intoxicated rats. J Biomed Sci 12: 185-195, 2005.

18. Lee TY, Wang GJ, Chiu JH and Lin HC: Long-term administration of Salvia miltiorrhiza ameliorates carbon tetrachloride-induced hepatic fibrosis in rats. J Pharm Pharmacol 55: 1561-1568, 2003.

19. Yang Y, Yang S, Chen M, Zhang X, Zou Y and Zhang X: Compound Astragalus and Salvia miltiorrhiza extract exerts anti-fibrosis by mediating TGF-beta/Smad signaling in myofibroblasts. J Ethnopharmacol 118: 264-270, 2008

20. Maeda K, Koda M, Matono T, Sugihara T, Yamamoto S, Ueki M, Murawaki Y, Yamashita N and Nishiyama S: Preventive effects of ME3738 on hepatic fibrosis induced by bile duct ligation in rats. Hepatol Res 38: 727-735, 2008.

21. Geerts A: History, heterogeneity, developmental biology, and functions of quiescent hepatic stellate cells (Review). Semin Liver Dis 21: 311-335, 2001.

22. Bataller R and Brenner DA: Hepatic stellate cells as a target for the treatment of liver fibrosis (Review). Semin Liver Dis 21: 437-451, 2001
23. Friedman SL: Molecular regulation of hepatic fibrosis, an integrated cellular response to tissue injury (Review). J Biol Chem 275: 2247-2250, 2000

24. Arthur MJ: Fibrogenesis II: Metalloproteinases and their inhibitors in liver fibrosis (Review). Am J Physiol Gastrointest Liver Physiol 279: G245-G249, 2000.

25. Chor JS, Yu J, Chan KK, Go YY and Sung JJ: Stephania tetrandra prevents and regresses liver fibrosis induced by carbon tetrachloride in rats. J Gastroenterol Hepatol 24: 853-859, 2009.

26. Tao LL, Cheng YY, Ding D, Mei S, Xu JW, Yu J, Ou-Yang Q, Deng L, Chen Q, Li QQ, et al: C/EBP- $\alpha$ ameliorates $\mathrm{C}\left(\mathrm{Cl}_{4}\right)$-induced liver fibrosis in mice through promoting apoptosis of hepatic stellate cells with little apoptotic effect on hepatocytes in vitro and in vivo. Apoptosis 17: 492-502, 2012.

27. Wang X, Ikejima K, Kon K, Arai K, Aoyama T, Okumura K, Abe W, Sato N and Watanabe S: Ursolic acid ameliorates hepatic fibrosis in the rat by specific induction of apoptosis in hepatic stellate cells. J Hepatol 55: 379-387, 2011.

28. Murata T, Naomoto Y, Yamatsuji T, Okawa T, Shirakawa Y, Gunduz M, Nobuhisa T, Takaoka M, Sirmali M, Nakajima M, et al: Localization of FAK is related with colorectal carcinogenesis. Int J Oncol 32: 791-796, 2008.

29. Xia J, Lv N, Hong Y, Li C, Tao X, Chen X and Cheng B: Increased expression of focal adhesion kinase correlates with cellular proliferation and apoptosis during 4-nitroquinoline-1oxide-induced rat tongue carcinogenesis. J Oral Pathol Med 38: 524-529, 2009.

30. Liu G, Meng X, Jin Y, Bai J, Zhao Y, Cui X, Chen F and Fu S: Inhibitory role of focal adhesion kinase on anoikis in the lung cancer cell A549. Cell Biol Int 32: 663-670, 2008.

31. Lewis TS, Shapiro PS and Ahn NG: Signal transduction through MAP kinase cascades. Adv Cancer Res 74: 49-139, 1998.

32. Karreth FA and Tuveson DA: Modelling oncogenic Ras/Raf signalling in the mouse. Curr Opin Genet Dev 19: 4-11, 2009.

33. Secker GA, Shortt AJ, Sampson E, Schwarz QP, Schultz GS and Daniels JT: TGF $\beta$ stimulated re-epithelialisation is regulated by CTGF and Ras/MEK/ERK signalling. Exp Cell Res 314: 131-142, 2008.

34. Ma FY, Sachchithananthan M, Flanc RS and Nikolic-Paterson DJ: Mitogen activated protein kinases in renal fibrosis (Review). Front Biosci (Schol Ed) 1: 171-187, 2009.

35. Smart DE, Green K, Oakley F, Weitzman JB, Yaniv M, Reynolds G, Mann J, Millward-Sadler H and Mann DA: JunD is a profibrogenic transcription factor regulated by Jun N-terminal kinase-independent phosphorylation. Hepatology 44: 1432-1440, 2006.

36. Qiang H, Lin Y, Zhang X, Zeng X, Shi J, Chen YX, Yang MF, Han ZG and Xie WF: Differential expression genes analyzed by cDNA array in the regulation of rat hepatic fibrogenesis. Liver Int 26: $1126-1137,2006$

37. Shen JG, Zhang XL and Huo XX: The role of FAK-ERK signal transduction pathway in apoptosis of hepatic stellate cell. Zhonghua Gan Zang Bing Za Zhi 16: 849-853, 2008 (In Chinese).

38. Shen JG, Zhang XL, Huo XX and Wei J: The role of focal adhesion kinase-extracellular signal regulated kinase signal transduction pathway in proliferation of hepatic stellate cell. J Gastroenterol Hepatol 21: A374, 2006.

39. An J, Zheng L, Xie S, Dun Z, Hao L, Yao D, Shih DQ and Zhang X: Down-regulation of focal adhesion kinase by short hairpin RNA increased apoptosis of rat hepatic stellate cells. APMIS 119: 319-329, 2011. 